\title{
Generalized nonlinear constitutive law applied to steel trapezoidal sheet plates
}

\author{
N. Staszak \\ $R \&$ D Department, FEMat sp. z o.o., Poznań, Poland \\ T. Gajewski \\ Institute of Structural Analysis, Poznan University of Technology, Poznań, Poland \\ T. Garbowski \\ Department of Biosystems Engineering, Poznan University of Life Sciences, Poznań, Poland
}

\begin{abstract}
In the paper, a modified nonlinear finite element method for analysis of trapezoidal plates geometrically reduced to shallow-shell Reissner-Mindlin formulation is presented. Due to the method proposed the complex plate cross-section and nonlinear materials may be modelled and no implementation of advanced constitutive law via user subroutines is needed. The generalized nonlinear constitutive law is used to update the stiffness of the plate element. The method enables modeling of complicated cross-sections, such as steel trapezoidal sheets, metal facing sandwich panels or reinforced concrete. Additionally, for those geometrically complex sections an advanced nonlinear material may be adopted. To verify the proposed method, a selected trapezoidal sheets were modeled in a commercial software as full 3D shell structures. By comparing displacements and forces, it was shown that both models behave almost identically, however, the simplified model has about 300-400 times less degrees of freedom, thus it is much more efficient.
\end{abstract}

\section{INTRODUCTION}

Steel trapezoidal plates are common structures used in civil engineering to cover roofs of industrial buildings. Such structures are easy to produce, transport and install at the building site. Due to its specific structure, they are light and have a very high ratio of load capacity to its mass. They are cheap, both as the material itself and in installation, and especially profitable, if there is a large plain roof area to cover. Its main disadvantage is low acoustic insulation, however, this is usually not the problem for industrial buildings such as warehouses or production halls.

Typical designing of such structures is easy due to detailed technical catalogues with load capacity tables included and released by its producers. However, for deeper strength analysis of the whole system of roofing, namely, steel trapezoidal sheets, bearing beams, roof windows, inspection holes, etc., such a simplified approach seems to be useless. Also, the formulas according to international standards, such as PN-EN 1993-1-3: 2008 - Eurocode 3 are pointless to apply. Moreover, if one would like to take into account a nonlinear aspect of steel or any other material in the system, only the detailed numerical model, for example utilizing a finite element method may give the reliable answer.

On the other hand, simulating the full geometry of the trapezoidal sheets may lead to a computationally very expensive models. In the recent 2020 paper of Zakhimi et al., one may find differentiation on three generations of sheeting, namely, unstiffened (see Figure 1a), 
a)

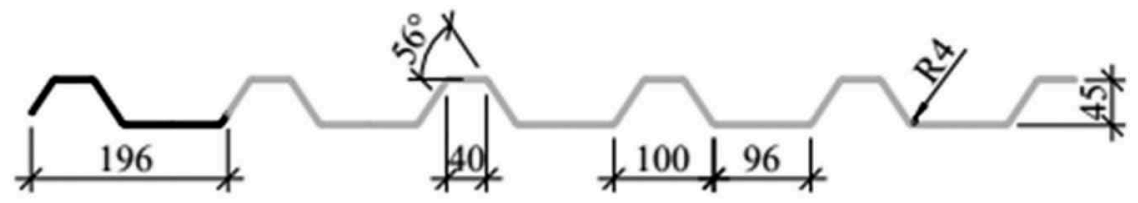

b)

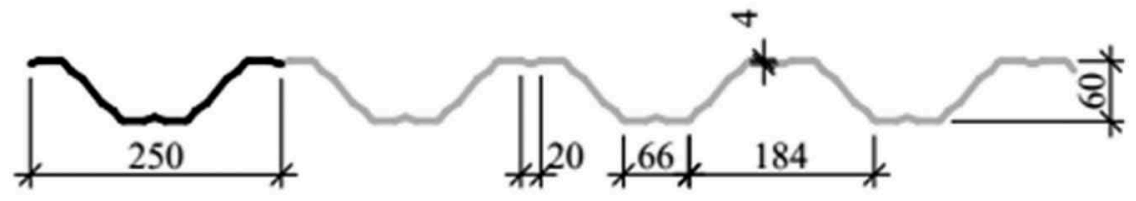

c)

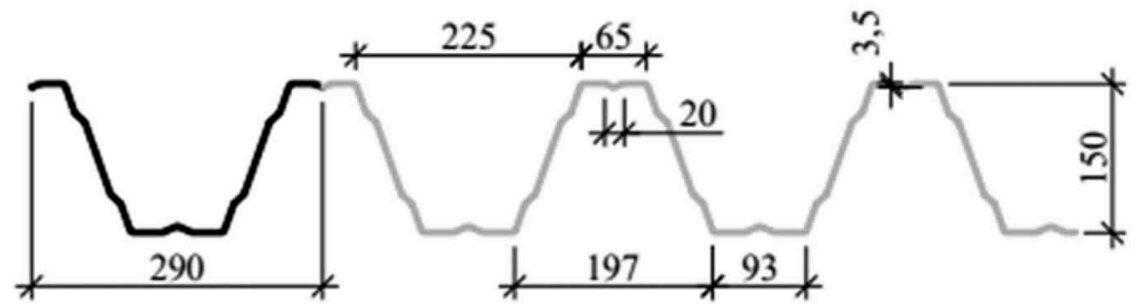

Figure 1. Examples of cross-sections of steel trapezoidal sheets used in civil engineering: a) T45 $(\mathrm{h}=$ $45 \mathrm{~mm}), \mathrm{b}) \mathrm{T} 60(\mathrm{~h}=60 \mathrm{~mm})$ and c) $\mathrm{T} 150(\mathrm{~h}=150 \mathrm{~mm})$.

longitudinally stiffened (see Figure 1b-c), and longitudinally and transversely stiffened. Stiffening is obtained by embossing the sheets. Steel sheets have not only simple idealized trapezoidal shapes, like in Figure 1a, but also more challenging shapes with embossing in two directions to model, see Figure 1b-c. Those profiling sheets due to its additional longitudinal and transverse stiffness are also in common use. The examples of detailed modelling of trapezoidal sheets are for instance available in the papers of Franco \& Batista (2017) or Flodr et al. (2014). Franco \& Batista analyzed what should be the outlines for longitudinal stiffeners called also intermediate stiffeners, in order to maximize the strength and economical objectives. Flodr et al. (2014) considered several numerical models with progressive complexity of modeling. In the paper, in each case the full geometry of trapezoidal sheets was used. As shown, this leads to computationally expensive models. The analytic approach, like the one shown by Mohammadi et al. (2015), is an alternative way to model steel trapezoidal sheets; this approach is not as versatile as the computational approach.

The objective of this paper is to introduce the computational approach to simplify the modelling of the full geometry of trapezoidal sheets in order to obtain a faster and comparably accurate model of finite element method. Finite element method is currently the leading method used for strength analysis of structures. Thus, the proposed modification may be easily implemented in common engineering softwares, without the need of advanced knowledge of finite element special techniques or nonlinear material modelling. The approach utilized in this paper is called the generalized nonlinear constitutive law (GNCL). In the literature, for instance, it was applied to model beams and frame structures as an attractive alternative of modelling full 3D geometries and nonlinear complex constitutive laws of steel or concrete in composite structures, see Łodygowski (1982), Łodygowski \& Szumigała (1992) and Szumigała (2007). In the paper of Mrówczyński et al. (2021), among others, the method was updated with the possibility of including shear effects (Timoshenko theory). The aim of this paper is to apply the GNCL method to model plate structures, in particular to effectively model steel trapezoidal sheets. The concept is original and was not applied before for plate structures. Otherwise the method is easy to use, if one would like to take into consideration in a simple plate modelling not only a complex geometry, but also material nonlinearities of steel instead of building an advanced and computationally expensive FEM model. 


\section{METHODS AND MATERIALS}

In Figure 2a, the overall algorithm of the proposed method is presented. After defining the input data (i.e. node coordinates, boundary conditions and cross-sections), the node displacements and internal forces are computed by using the finite element method. Based on the nodal displacements, the strains (normal strains $\boldsymbol{\varepsilon}_{0}$, shear strains $\gamma$ and curvature $\boldsymbol{\kappa}$ ) are computed, which makes it possible to determine the reduced stiffness of each element. For new stiffnesses, the displacements are computed and subsequent iterations are carried out until the increment of structure displacements is smaller than the assumed tolerance.

In Figure 2b, the stiffness reduction function begins with the division of the cross-section into smaller layers for which the areas and position are determined. Based on the strains determined in the main part of the algorithm, the reduced strains in each layer are determined, and then the reduced stresses, element stiffnesses and the position of the neutral axis are computed. Successive iterations are performed until the change of the position of the neutral axis is smaller than the assumed tolerance. The mathematical formulation of the method proposed may be found in Mrówczyński et al. (2021).

Reissner-Mindlin plate is considered in this paper. Assuming the $x y z$ coordinate system, the displacement field for plates reads:

$$
\begin{gathered}
u(x, y, z)=z \theta_{x}(x, y), \\
v(x, y, z)=z \theta_{y}(x, y), \\
w(x, y, z)=w_{0}(x, y),
\end{gathered}
$$

a)

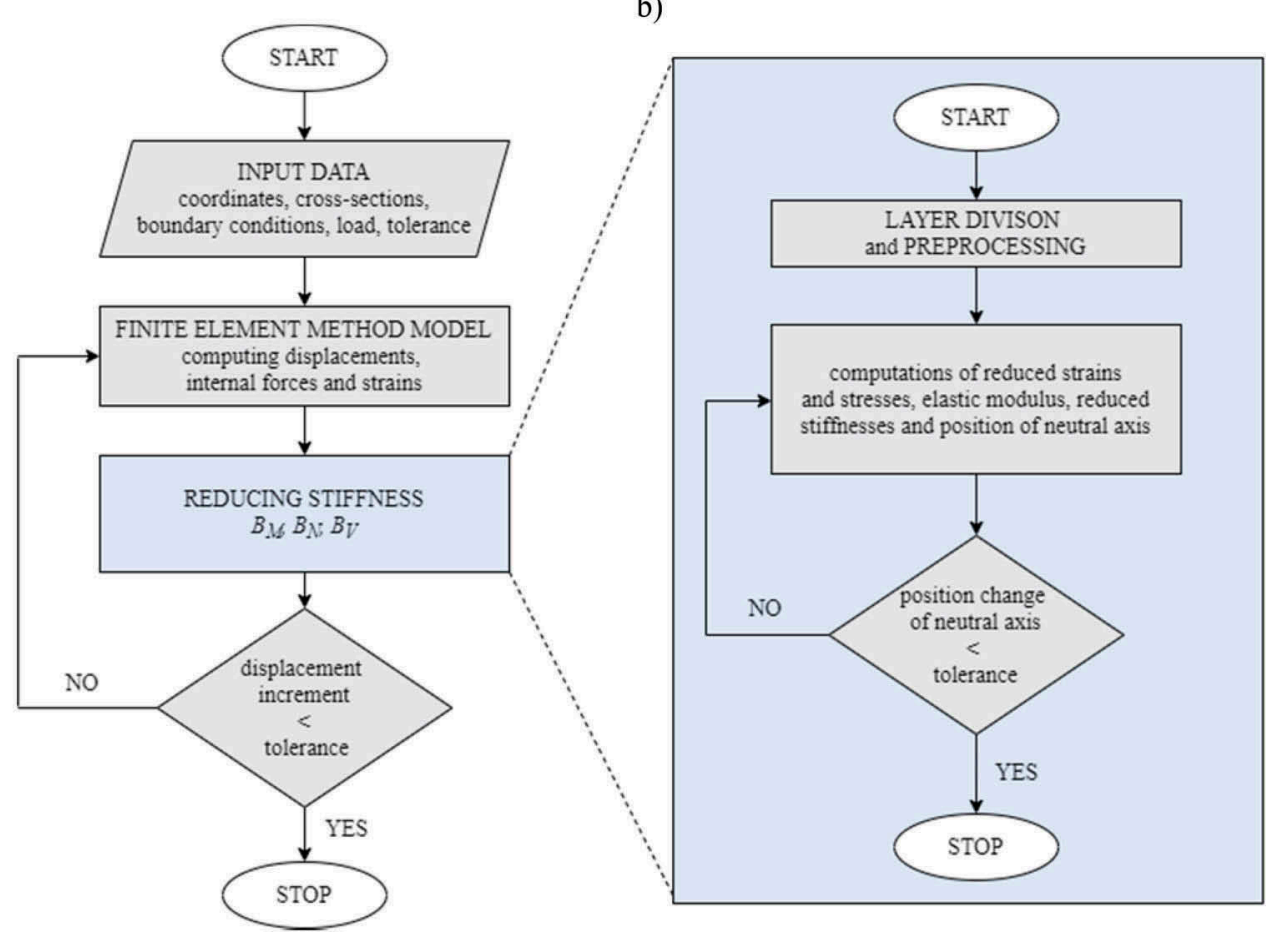

Figure 2. The GNCL algorithm: a) the overall scheme and b) the stiffness reduction function (where $B_{M}$ is bending stiffness. $B_{N}$ is tensile stiffness and $B_{V}$ is shear stiffness). 
where $u, v$ are two in-plane mid-surface displacements, while $w$ is out of plane mid-surface displacement of the plate; $\theta_{x}$ and $\theta_{y}$ are two rotations of the normal on the plane $x z$ and $y z$ :

$$
\theta_{x}=\partial w / \partial x+\phi_{x} ; \theta_{y}=\partial w / \partial y+\phi_{y}
$$

noting that $\gamma_{x}=-\phi_{x}$ and $\gamma_{y}=-\phi_{y}$.

In both the $x z$ and $y z$ vertical planes, the normal rotation was obtained as the sum of two rotations: (i) the corresponding slope of the middle plane of the plate and (ii) the additional rotation $\phi$, which results from the lack of orthogonality of the normal to the middle plane after deformation. Therefore, the rotations $\theta_{x}$ and $\theta_{y}$ cannot be calculated from the deflection only and become independent variables. This is the fundamental difference between ReissnerMindlin and Kirchhoff-Love plate theories. Relationships between strains (membrane $-\boldsymbol{\varepsilon}$, bending $-\boldsymbol{\kappa}$ and shear $-\boldsymbol{\gamma}$ ) and displacements are:

$$
\begin{gathered}
\epsilon=\boldsymbol{\varepsilon}+z \mathbf{\kappa}, \\
\boldsymbol{\varepsilon}=\left[\begin{array}{c}
\varepsilon_{x} \\
\varepsilon_{y} \\
\gamma_{x y}
\end{array}\right]=\left[\begin{array}{c}
\partial u / \partial x \\
\partial v / \partial y \\
\partial u / \partial y+\partial v / \partial x
\end{array}\right]=-z\left[\begin{array}{c}
\partial \theta_{x} / \partial x \\
\partial \theta_{y} / \partial y \\
\partial \theta_{x} / \partial y+\partial \theta_{y} / \partial x
\end{array}\right], \\
\mathbf{\kappa}=\left[\begin{array}{c}
\kappa_{x} \\
\kappa_{y} \\
\kappa_{x y}
\end{array}\right]=-\left[\begin{array}{c}
\partial \theta_{x} / \partial x \\
\partial \theta_{y} / \partial y \\
\partial \theta_{x} / \partial y+\partial \theta_{y} / \partial x
\end{array}\right]=-\left[\begin{array}{c}
\partial^{2} w / \partial x^{2} \\
\partial^{2} w / \partial y^{2} \\
\partial^{2} w / \partial x \partial y
\end{array}\right], \\
\boldsymbol{\gamma}=\left[\begin{array}{c}
\gamma_{x z} \\
\gamma_{x y}
\end{array}\right]=\left[\begin{array}{c}
\partial w / \partial x+\partial u / \partial z \\
\partial w / \partial y+\partial v / \partial z
\end{array}\right]=\left[\begin{array}{c}
\partial w / \partial x-\theta_{x} \\
\partial w / \partial y-\theta_{y}
\end{array}\right]=\left[\begin{array}{c}
-\phi_{x} \\
-\phi_{y}
\end{array}\right] .
\end{gathered}
$$

The stress-strain relations in local coordinates are given by:

$$
\begin{aligned}
& {\left[\begin{array}{c}
\sigma_{x} \\
\sigma_{y} \\
\sigma_{x y}
\end{array}\right]=\frac{E}{1-\nu^{2}}\left[\begin{array}{ccc}
1 & \nu & 0 \\
\nu & 1 & 0 \\
0 & 0 & \frac{1-v}{2}
\end{array}\right]\left[\begin{array}{c}
\varepsilon_{x} \\
\varepsilon_{y} \\
\gamma_{x y}
\end{array}\right],} \\
& \left\{\begin{array}{l}
\sigma_{x z} \\
\sigma_{y z}
\end{array}\right\}=\frac{k E}{2(1+\nu)}\left[\begin{array}{ll}
1 & 0 \\
0 & 1
\end{array}\right]\left\{\begin{array}{l}
\gamma_{x z} \\
\gamma_{y z}
\end{array}\right\},
\end{aligned}
$$

where $k$ is a shear correction factor, or in the compact form

$$
\boldsymbol{\sigma}=\mathbf{C} \in, \boldsymbol{\tau}=\mathbf{C}_{s} \boldsymbol{\gamma}
$$

The strain energy, $U$, reads

$$
U=\frac{1}{2} \int_{A}\left(\in^{T} \boldsymbol{D}^{*} \in+\boldsymbol{\gamma}^{T} \boldsymbol{D}_{s}^{*} \boldsymbol{\gamma}\right) d A
$$

where

$$
\boldsymbol{D}^{*}=\left[\begin{array}{ll}
\boldsymbol{A} & \boldsymbol{B} \\
\boldsymbol{B} & \boldsymbol{D}
\end{array}\right], \boldsymbol{D}_{s}^{*}=\boldsymbol{D}_{s}
$$

and $\mathbf{A}, \mathbf{B}, \mathbf{D}$ and $\mathbf{D}_{s}$ are stiffnesses of the plates given by: 
Table 1. Cross-section characteristics of trapezoidal sheets in $x-z$ plane.

\begin{tabular}{lllll}
\hline \multirow{2}{*}{ Cross-section } & Area & Inertia moment & Sheet thickness & Neutral axis position \\
\cline { 2 - 5 } & {$\left[\mathrm{cm}^{2}\right]$} & {$\left[\mathrm{cm}^{4}\right]$} & {$[\mathrm{mm}]$} & {$[\mathrm{cm}]$} \\
\hline T45 & 1.214 & 3.365 & 0.50 & 2.798 \\
T60 & 1.516 & 8.285 & 0.50 & 3.029 \\
T150 & 4.884 & 144.397 & 1.00 & 7.927 \\
\hline
\end{tabular}

$$
\begin{aligned}
(\mathbf{A}, \mathbf{B}, \mathbf{D}) & =\frac{1}{P} \iint \mathbf{C}\left(1, z, z^{2}\right) d x d z, \\
\mathbf{D}_{s} & =\frac{1}{P} \iint \mathbf{C}_{s} d x d z
\end{aligned}
$$

where $P$ is a wavelength period. For the sake of simplicity, the same stiffness in the $y$ direction was used, which is obviously a false assumption, but does not disturb the solution of the considered here a problem of a unidirectional bending of the plate.

If the matrix $\mathbf{B}$ becomes $\mathbf{0}$ then the membrane and bending effects are uncoupled and the neutral plane coincided with the plane $x y$. This means that the bending moments did not cause any membrane strains and the normal forces did not cause any curvature.

\section{COMPUTATIONAL EXAMPLES}

Here, three examples of a trapezoidal sheet with the cross-sections presented in Figure 1 are considered. The material parameters used in the reference model were selected in accordance with the specifications provided by the manufacturer. The initial sheet stiffnesses were calculated on the basis of equations (14) and (15), the characteristics used to compute $\mathbf{A}, \mathbf{B}, \mathbf{D}$ and $\mathbf{D}_{s}$ matrices are presented in Table 1.

The reference models were built and calculated in Abaqus using S4 shell elements with 4 nodes and 6 degrees of freedom at each node. The number of finite elements used in each model was selected to accurately reproduce the cross-sectional geometry of the trapezoidal sheet and was equal to $15,678,23,450,22,244$ for T45, T60 and T150, respectively. A static nonlinear displacement control analysis was used to generate force-displacement curves for later comparison with the curves obtained from the proposed algorithm described in Section 2, using simple shallow plate elements and GNCL.

\section{COMPUTATIONAL RESULTS}

In Figure 3, the deformed geometry of three trapezoidal sheets are presented, which were obtained from the full geometry reference models. The GNCL algorithm ensures the correct mapping of displacements, the stresses are calculated separately to reduce the equivalent stiffness. Later, only the section forces and moments are available.

Figure 4 shows a comparison of the force-displacement curves obtained from the reference models and from the simplified approach based on a simple shallow plate finite element (50 elements) and the GNCL algorithm described in Section 2. It should be noted that the number of elements used in the simplified (GNCL) models was about 300-400 times smaller than the number of elements used in the reference models.

Figures 3 and 4 show that the simplified methods presented here give results that both qualitatively and quantitatively do not differ from the results obtained from the reference 
a)

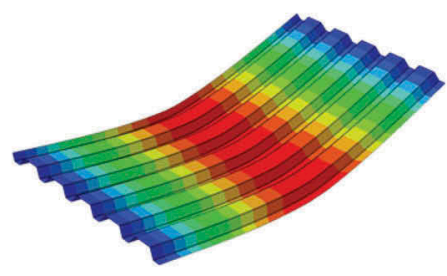

b)

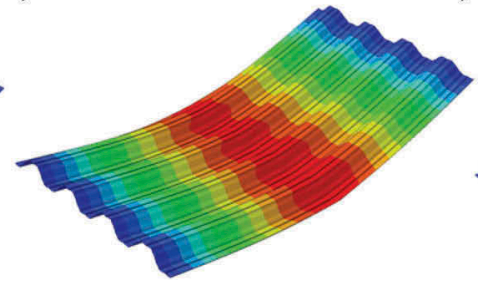

c)

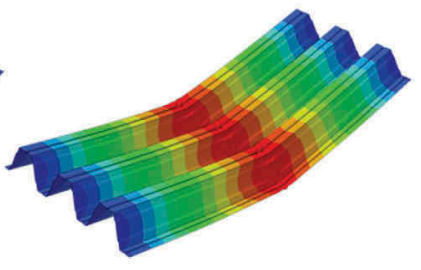

Figure 3. Displacement fields of finite element reference models for a) T45 (15678 elements), b) T60 (23450 elements) and c) T150 (22244 elements).

a)

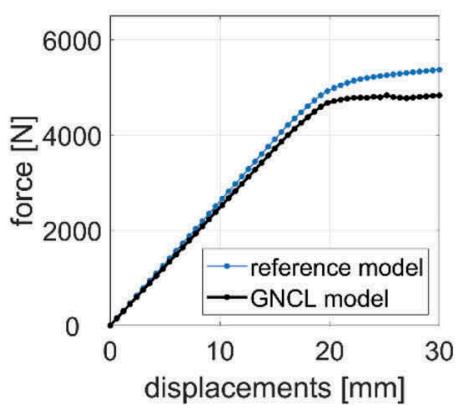

b)

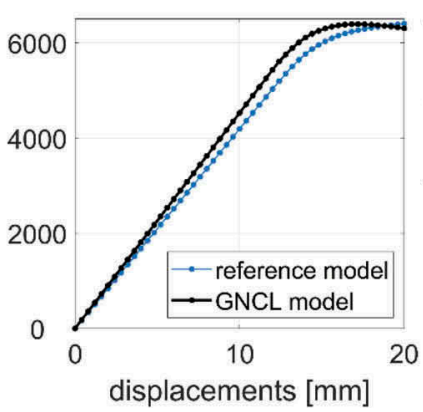

c)

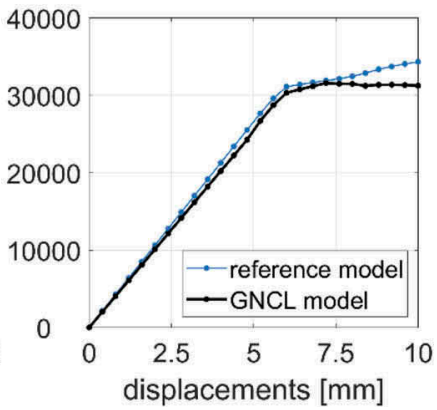

Figure 4. Force vs. displacement plots for trapezoidal sheet of a) T45, b) T60 and c) T150.

models. The difference between the maximal values of force is not greater than $10 \%$, what is acceptable in civil structures, in which the safety factors are much greater. This shows that the approach proposed here can be successfully applied to such calculations.

\section{CONCLUSIONS}

In the paper, the generalized nonlinear constitutive law was applied to steel trapezoidal sheet plates. The comparison between the modelling of full geometry of trapezoidal sheets and simplified approach of GNCL algorithm was presented for three trapezoidal sheets commonly used by designers of such structures. As shown, using the full geometry leads to computationally expensive models. On the contrary, the GNCL method not only, enables faster and similar accurate computations, but also enables, utilizing the material nonlinearities.

\section{REFERENCES}

Flodr J., Krejsa M., Mikolasek D., Sucharda O. \& Zidek L. 2014. Mathematical Modelling of Thin-Walled Cold-Rolled Cross-Section. Applied Mechanics and Materials 617: 171-174.

Juarez Moara Santos Franco \& Eduardo de Miranda Batista 2017. Buckling behavior and strength of thin-walled stiffened trapezoidal CFS under flexural bending. Thin-Walled Structures 117: $268-281$.

Łodygowski, T. 1982. Geometrycznie nieliniowa analiza sztywno-plastycznych i sprężysto-plastycznych belek i ram płaskich. Warsaw.

Łodygowski, T. \& Szumigała, M. 1992. Engineering models for numerical analysis of composite bending members. Mechanics of Structures and Machines 20: 363-380. 
Mohammadi H., Ziaei-Rad S. \& Dayyani I., 2015. An equivalent model for trapezoidal corrugated cores based on homogenization method. Composite Structures 131: 160-170.

Mrówczyński D., Gajewski T. \& Garbowski T., 2021, Application of the generalized nonlinear constitutive law in 2D shear flexible Beam Structures, Archive of Civil Engineering (accepted for publication).

PN-EN 1993-1-3: 2008 - Eurocode 3: Design of steel structures - Part 1-3: General rules - Supplementary rules for cold-formed members and sheeting, 2008.

Szumigała, M. 2007. Zespolone stalowo-betonowe konstrukcje szkieletowe pod obciążeniem doraźnym. Wydawnictwo Politechniki Poznańskiej. Poland.

Trapezoidal tables for sheets T45/196, T60/250 and T150/290. Technical catalogue for designers. Blachy Pruszyński. [https://pruszynski.com.pl/catalogue.php, online access: 5/11/2020].

Zakhimi H., Hofmeyer H., Snijder H.H, \& Mahendran M. 2020. Explicit and interaction direct strength methods for combined web crippling and bending moment failure of first-generation trapezoidal steel sheeting. Thin-Walled Structures 157. 